\title{
How Secure is Deterministic Encryption?
}

\author{
Mihir Bellare ${ }^{1(\bowtie)}$, Rafael Dowsley ${ }^{2}$, and Sriram Keelveedhi ${ }^{1,2}$ \\ 1 Department of Computer Science and Engineering, \\ University of California San Diego, San Diego, USA \\ mihir@eng.ucsd.edu \\ 2 Institute of Theoretical Informatics, Karlsruhe Institute of Technology, \\ Karlsruhe, Germany
}

\begin{abstract}
This paper presents three curious findings about deterministic public-key encryption (D-PKE) that further our understanding of its security, in particular because of the contrast with standard, randomized public-key encryption (R-PKE):

- It would appear to be a triviality, for any primitive, that security in the standard model implies security in the random-oracle model, and it is certainly true, and easily proven, for R-PKE. For D-PKE it is not clear and depends on details of the definition. In particular we can show it in the non-uniform case but not in the uniform case.

- The power of selective-opening attacks (SOA) comes from an adversary's ability, upon corrupting a sender, to learn not just the message but also the coins used for encryption. For R-PKE, security is achievable. For D-PKE, where there are no coins, one's first impression may be that SOAs are vacuous and security should be easily achievable. We show instead that SOA-security is impossible, meaning no D-PKE scheme can achieve it.

- For R-PKE, single-user security implies multi-user security, but we show that there are D-PKE schemes secure for a single user and insecure with two users.
\end{abstract}

\section{Introduction}

Public-key encryption (PKE) schemes are usually randomized, in order to achieve goals like IND-CPA [29]. BBO [5] introduced deterministic PKE (DPKE), arguing that it offers practical benefits over randomized PKE (R-PKE) in certain applications. These include efficient search on encrypted databases [5] and resilience in the face of the low-quality randomness that pervades systems $[6,41] .^{1}$

BBO [5] provide a definition PRIV of "best possible" security for D-PKE, and ROM constructions achieving it. Equivalent, IND-style formulations appear in [10]. These definitions are unusual, and achieving them in the standard model

\footnotetext{
${ }^{1}$ Weak randomness leads to catastrophic failures in R-PKE including the ability to recover the plaintext from the ciphertext in schemes including GM, El Gamal and Rabin-SAEP [36].

(C) International Association for Cryptologic Research 2015

J. Katz (Ed.): PKC 2015, LNCS 9020, pp. 52-73, 2015.

DOI: $10.1007 / 978-3-662-46447-2 \_3$
} 
(SM) is challenging. Emerging as a practically-motivated notion of theoretical depth and interest, D-PKE has attracted significant foundational work as researchers aim to understand the properties and achievability of the basic definitions and variants $[10,11,19,20,28,33,39]$. We continue this line of work.

OUR WORK. This paper shows that determinism impacts security in beyondobvious ways. Specifically, we consider three questions. The first is whether security in the standard model implies security in the ROM. The second is whether D-PKE is secure under selective-opening attack. The last is whether single-user security implies multi-user security. Fig. 1 summarizes our findings, which are discussed in more depth below. On the practical side, our work indicates that care must be taken in the use of D-PKE. On the theoretical side it indicates further foundational subtleties for D-PKE, and, more broadly, for multi-stage security definitions, in the wake of those already indicated in [40,42].

BACKGROUND. In R-PKE, the encryption algorithm Enc takes the public (encryption) key $p k$, message $m$ and coins $r$ to return a ciphertext $c=\operatorname{Enc}(p k, m ; r)$. The basic notion of security is IND-CPA $[7,29]$. An adversary is a pair $\left(A_{1}, A_{2}\right)$ of PT algorithms. The game picks keys $(p k, s k)$ and a challenge bit $b$. We run $A_{1}$ on input $p k$ to get a pair $\left(m_{0}, m_{1}\right)$ of messages and state information st. The game picks random coins $r$, computes challenge ciphertext $c=\operatorname{Enc}\left(p k, m_{b} ; r\right)$ and runs $A_{2}$ on $c$, st to get a bit $b^{\prime}$. Security requires that $2 \operatorname{Pr}\left[b=b^{\prime}\right]-1$ is negligible.

In D-PKE [5], there are no coins, Enc taking $p k, m$ to return $c=\operatorname{Enc}(p k, m)$. Such a scheme cannot achieve IND-CPA. The notion we use is IND [10], an indistinguishability variant of the PRIV notion of [5]. An adversary is a pair $\left(A_{1}, A_{2}\right)$ of $\mathrm{PT}$ algorithms. The game picks keys $(p k, s k)$ and a challenge bit $b$. We run $A_{1}$ (it does not get $p k$ ) to get a pair $\left(\mathbf{m}_{0}, \mathbf{m}_{1}\right)$ of vectors of messages (but no state information). The game computes challenge ciphertext vector $\mathbf{c}=$ $\operatorname{Enc}\left(p k, \mathbf{m}_{b}\right)$, encryption being component-wise, and runs $A_{2}$ on $\mathbf{c}, p k$ to get a bit $b^{\prime}$. Security requires that $2 \operatorname{Pr}\left[b=b^{\prime}\right]-1$ is negligible. Important restrictions are that (1) $A_{1}$ does not get the public key (2) each individual message in the vectors $\mathbf{m}_{0}, \mathbf{m}_{1}$ has high min-entropy, meaning is statistically unpredictable, and (3) $A_{1}, A_{2}$ do not communicate directly, meaning no state information is passed from $A_{1}$ to $A_{2}$. These restrictions are necessary, for without them security is not achievable.

In the ROM [14], both stages of the adversary have access to the random oracle RO, whether for R-PKE or D-PKE. In the latter case, the min-entropy condition is required to hold even given (conditioned on) the RO.

Does SM-SECURITy IMPLy ROM-SECURITy? That security in the standard model (SM) implies security in the ROM appears to be a triviality or tautology, true for any primitive. To be specific, suppose we have a standard-model R-PKE scheme, meaning the algorithms of the scheme make no calls to RO. Suppose it is IND-CPA in the SM. Then it is IND-CPA in the ROM. Intuitively this seems clear because if the scheme does not use the RO, then giving the adversary access to $\mathrm{RO}$ cannot violate security. If we want to prove the claim formally, we could do so by reduction. Given a ROM adversary $\left(A_{1}, A_{2}\right)$, we build SM adversary 
$\left(B_{1}, B_{2}\right)$ with the same advantage by just having $B_{1}$ and $B_{2}$ simulate the RO. Thus, $B_{1}$ maintains a table $H$, and runs $A_{1}$. When the latter makes a query $\mathrm{RO}(x)$, adversary $B_{1}$ checks if $H[x]$ is defined, and, if not, picks it at random, in either case returning $H[x]$ to $A_{1}$ as the answer. When $A_{1}$ halts with output $\left(m_{0}, m_{1}\right)$ and state $\mathrm{st}_{A}$, adversary $B_{1}$ halts with output $\left(m_{0}, m_{1}\right)$ and state st ${ }_{B}$, where the latter consists of $\mathrm{st}_{A}$ plus the populated (defined) part of table $H$, which has polynomial size. Now $B_{2}$, given $c$, st ${ }_{B}$, runs $A_{2}\left(c, \mathrm{st}_{A}\right)$, continuing to respond to $A_{2}$ 's oracle queries via table $H$, suitably augmenting it as necessary for new queries. Eventually $B_{2}$ returns whatever $A_{2}$ returns. It is clear that SM adversary $\left(B_{1}, B_{2}\right)$ simulates ROM adversary $\left(A_{1}, A_{2}\right)$ perfectly and has the same advantage.

The claim that SM security implies ROM security, and the simulation argument above to establish it, hardly seem specific to R-PKE. It would appear to be true that SM security trivially implies ROM security for any primitive via such an argument.

But for D-PKE, the argument fails, and whether SM security implies ROM security is not clear. To see why, let us try to mimic the above argument for DPKE. We can design $B_{1}$, simulating $A_{1}$, in the same way. The difficulty is that $B_{1}$ cannot pass its partial table $H$ to $B_{2}$, for no state information is allowed to flow from $B_{1}$ to $B_{2}$. This leaves $B_{2}$ stuck. It could simulate a new RO for $A_{2}$, but in the real ROM game, $A_{1}, A_{2}$ see the same RO, not different ones. The question this raises is whether the difficulty is inherent, meaning SM security does not imply ROM security, or whether some alternative argument can show the implication.

We find that the answer depends on details of the definition. Let $\mathrm{IND}_{\mathrm{u}}, \mathrm{IND}_{\mathrm{nu}}$ denote, respectively, the uniform and non-uniform renditions of IND. That is, in the first case, the adversaries are TMs while in the second they are families of circuits. We show that SM security implies ROM security for $\mathrm{IND}_{\mathrm{nu}}$. Our proof works by starting with ROM adversaries $\mathrm{A}_{1}, \mathrm{~A}_{2}$, hardwiring a $q(\cdot)$-wise independent hash function $h$ into the circuits of $B_{1}, B_{2}$, and having these circuits use $h$ to simulate RO for $A_{1}, A_{2}$, with $q(\cdot)$ depending on the number of oracle queries of $A_{1}$ and $A_{2}$. We show that there exists a "good" choice of $h$ under which the simulation is correct. However, in the case of $\mathrm{IND}_{\mathrm{u}}$, we were not able to settle the question. That is, we see no way to prove that SM security implies ROM security (it is not clear how to perform a simulation, and it is not clear there is any other approach to the proof) but nor can we imagine a counterexample (it would need to exploit the fact that the scheme is secure for uniform adversaries but not for non-uniform ones, for otherwise the claim is true).

Intuitively, it is hard for us to imagine how a SM scheme can be insecure in the ROM, meaning how an adversary can exploit the RO when scheme algorithms do not even use it. ${ }^{2}$ We found it curious that it was not obvious how to prove this and that it is not clear if it is even true in the uniform case.

${ }^{2}$ One might imagine the adversary gaining an advantage by having $B_{1}$ pick messages that depend on the RO in some clever way. The reason this does not appear to help the adversary is that each message is required to have high min-entropy even conditioned on the entire random oracle. 


\begin{tabular}{|c||c|c|c|}
\hline Primitive & SM $\Rightarrow$ ROM & SOA & SU $\Rightarrow$ MU \\
\hline \hline R-PKE & Yes & Yes & Yes \\
D-PKE & Sometimes & No & No \\
\hline
\end{tabular}

Fig. 1. Summary of our results: The first column indicates whether or not security in the standard model (SM) implies security in the ROM, the "sometimes" for DPKE reflecting that we can show it in the non-uniform case but not in the uniform case. The second column indicates whether or not security against selective-opening attack (SOA) is achievable. The third column indicates whether or not single-user (SU) security implies multi-user (MU) security.

These findings show further subtleties for multi-stage security definitions following ones already discovered by $[40,42]$, making D-PKE a central test case in this subtle and surprising domain.

Is SOA-SECURE D-PKE ACHIEVABLE? In a selective opening attack (SOA) on a R-PKE scheme, a vector $\mathbf{m}$ of $n$ messages is chosen from some distribution, a vector $\mathbf{r}$ of random and independent coins is chosen, and the adversary $A$ is given the ciphertext vector $\mathbf{c}=\operatorname{Enc}(p k, \mathbf{m} ; \mathbf{r}) . A$ responds with a subset $I$ of $\{1, \ldots, n\}$. In the message-only version of the attack, it is returned $\langle\mathbf{m}[i]$ : $i \in I\rangle$; in full SOA, it is returned both $\langle\mathbf{m}[i]: i \in I\rangle$ and $\langle\mathbf{r}[i]: i \in I\rangle$. In either case, to win, it has to compute some non-trivial information about $\langle\mathbf{m}[i]: i \notin I\rangle$. Security for the message-only version is implied by IND-CPA, as shown in [17], and is thus easily achievable. Security for full SOA is not implied by IND-CPA [9]. However, using lossy encryption [12,17,32,38], it is shown in $[12,17]$ that there exist schemes that provide full SOA under standard assumptions, so full SOA security is achievable, under standard assumptions in the standard model. Subsequently, further schemes have been provided as well $[27,30]$.

The question of security of D-PKE under SOA has not been considered before, and we initiate an investigation. A vector $\mathbf{m}$ of $n$ messages is again chosen from some distribution, and the adversary $A$ is given the ciphertext vector $\mathbf{c}=\operatorname{Enc}(p k, \mathbf{m}) . A$ responds with a subset $I$ of $\{1, \ldots, n\}$, is returned $\langle\mathbf{m}[i]: i \in I\rangle$, and, to win, has to compute some non-trivial information about $\langle\mathbf{m}[i]: i \notin I\rangle$. We note that what we have defined is the message-only version. Naturally, there is no "full" SOA here, since there are no coins used, and thus none to expose.

The difficulty of achieving SOA-secure R-PKE lies in exposure of the coins. Since D-PKE has no coins, one's first impression may be that SOA-security for it would be like message-only SOA-security for R-PKE and thus easy to achieve. To the contrary, we show that SOA-secure D-PKE is impossible. That is, there is no D-PKE scheme that is SOA-secure. Given any D-PKE scheme, we give an attack violating SOA-security.

The contrast with R-PKE is two-fold. For the latter, SOA is easy in the message-only case, and, with exposure of coins, even if not easy, it is achievable. But for D-PKE, it is simply not achievable. The key element of our proof is to 
show that for any D-PKE scheme there is an algorithm that can impose and verify an association between a message and ciphertext that is unique with high probability, even for dishonestly chosen public keys. We combine this with the technique of BDWY [9] to obtain our impossibility result. We note that for R-PKE the BDWY technique did not show impossibility of (full) SOA for all R-PKE schemes, but for a subclass of them, while we are using the technique to rule out SOA-security for all D-PKE schemes.

The problem of SOA-security has been the subject of many works $[2,3,12,16$ 18,21-24,26,27,30,31,34,37]. These have looked at R-PKE, commitment and IBE. We are the first to consider SOA for D-PKE.

Does SU SECURITy Imply MU SECURITY? The basic IND-CPA notion for RPKE [29] is a single-user (SU) setting, meaning there is only one public key in the game. In practice, many users, each with their own key pair, could encrypt messages, and these messages may be related. Security of R-PKE in the multiuser (MU) setting was defined in $[1,4]$. They showed that SU security implied MU security, meaning any R-PKE scheme that meets the usual SU IND-CPA notion is also MU secure.

It is natural to ask whether the same is true for D-PKE, namely whether SU security, in the form of IND, implies MU security. We define MU security for D-PKE and show that the answer to the question is "no." That is, we present a counter-example, namely a D-PKE scheme that we show meets the standard SU IND definition, but we give an attack showing that it fails to be MU-secure. Indeed, it is insecure even with just two users, meaning when there are two public keys in the picture.

BBO [5] had conjectured that indeed SU security did not in general imply MU security for D-PKE. Our results prove and confirm this conjecture. Brakerski and Segev [20] define MU security of D-PKE in the auxiliary input setting and give a scheme that achieves it for messages that are block sources, but they do not show a separation between the SU and MU settings. Dodis, Lee and Yum [25] give another example of a setting where SU security does not imply MU security, namely optimistic fair exchange.

\section{Preliminaries}

Notation And COnventions. We let $\lambda \in \mathbb{N}$ denote the security parameter. If $n \in \mathbb{N}$ then we let $1^{n}$ denote the string of $n$ ones and $[n]$ denote the set $\{1, \ldots, n\}$. If $A$ is a finite set, then let $|A|$ denote its size, and $a \stackrel{\$}{\leftarrow} A$ denote sampling $a$ uniformly at random from $A$. The empty string is denoted by $\varepsilon$. If $a$ and $b$ are two bit strings, we denote by $a \| b$ their concatenation. We use boldface letters for vectors. For any vector $\mathbf{x}$, we let $|\mathbf{x}|$ denote the number of its components. We say $\mathbf{x}$ is an $n$-vector if $|\mathbf{x}|=n$. For $i \in[|\mathbf{x}|]$ we let $\mathbf{x}[i]$ denote the $i$-th component of $\mathbf{x}$. We let $\operatorname{Maps}(D, R)$ denote the set of all functions $f: D \rightarrow R$.

Algorithms are randomized, unless otherwise specified as being deterministic. "PT" stands for "polynomial-time," whether for randomized algorithms 
or deterministic ones. If $A$ is an algorithm, we let $y \leftarrow A\left(x_{1}, \ldots ; r\right)$ denote running $A$ with random coins $r$ on inputs $x_{1}, \ldots$ and assigning the output to $y$. We let $y \stackrel{\$}{\leftarrow} A\left(x_{1}, \ldots\right)$ be the resulting of picking $r$ at random and letting $y \leftarrow A\left(x_{1}, \ldots ; r\right)$. We let $\left[A\left(x_{1}, \ldots\right)\right]$ denote the set of all $y$ that have positive probability of being output by $A\left(x_{1}, \ldots\right)$. A function $\epsilon: \mathbb{N} \rightarrow \mathbb{R}$ is negligible if for every polynomial $p$, there exists $\lambda_{p} \in \mathbb{N}$ such that $\epsilon(\lambda) \leq 1 / p(\lambda)$ for all $\lambda \geq \lambda_{p}$. An algorithm $A$ is uniform if there exists a Turing machine $T$ which halts with the output of $A$ on all inputs. An algorithm $A$ is non-uniform if there exists a sequence of circuits $\left\{C_{\lambda}\right\}_{\lambda \in \mathbb{N}}$ such that $C_{\lambda}$ computes $A\left(1^{\lambda}, \ldots\right)$.

Games. Our definitions and proofs use the code-based game-playing framework of [15] with some of the syntax of [40]. A game $G(\lambda)$ (see Fig. 2 for an example) consists of a MAIN procedure, and possibly others, and begins by executing MAIN, which runs an adversary $\mathrm{A}$ after some initialization steps. $\mathrm{A}$ is given oracle access to certain game procedures. After $A$ finishes executing, $G$ performs steps with $A$ 's output to produce some output itself. We assume that boolean variables are initialized to false, that sets are initialized to $\emptyset$, strings are initialized to $\epsilon$, and that integers are initialized to 0 . We denote by $G^{A} \Rightarrow y$ the event that an execution of $G$ with $A$ outputs $y$. We abbreviate $G^{A} \Rightarrow$ true as $G^{A}$.

Functions families. A family of functions $\mathrm{HF}$ is a PT, deterministic algorithm that defines for each $\lambda \in \mathbb{N}$ a map $\operatorname{HF}\left(1^{\lambda}, \cdot, \cdot\right):\{0,1\}^{\mathrm{HF} . \mathrm{kl}(\lambda)} \times\{0,1\}^{\mathrm{HF} . \mathrm{il}(\lambda)} \rightarrow$ $\{0,1\}^{\mathrm{HF} . o l(\lambda)}$. Here HF.kl, HF.il, HF.ol: $\mathbb{N} \rightarrow \mathbb{N}$ are the key, input and output lengths of $\mathrm{HF}$, respectively. We extend HF to vectors (in a component-wise way) via

$$
\mathrm{HF}\left(1^{\lambda}, k, \mathbf{x}\right)=\left(\mathrm{HF}\left(1^{\lambda}, k, \mathbf{x}[1]\right), \ldots, \mathrm{HF}\left(1^{\lambda}, k, \mathbf{x}[|\mathbf{x}|]\right)\right)
$$

for all $\lambda \in \mathbb{N}$, all $k \in\{0,1\}^{\mathrm{HF} . \mathrm{kl}(\lambda)}$ and all vectors $\mathbf{x}$ over $\{0,1\}^{\mathrm{HF} . \mathrm{il}(\lambda)}$.

\section{Deterministic PKE}

We provide definitions for D-PKE following [5,10]. We give a unified treatment of the ROM and the SM by regarding the latter as a special case of the former.

D-PKE. A deterministic public key encryption (D-PKE) scheme DE specifies four PT algorithms and related functions as follows. The parameter generator algorithm DE.Pg takes as input a unary representation $1^{\lambda}$ of the security parameter $\lambda \in \mathbb{N}$ and returns the system parameters $\pi \in\{0,1\}^{\mathrm{DE} . \mathrm{pl}(\lambda)}$ which are common to all users. The key generation algorithm DE.Kg takes as input $\pi$ and outputs a public encryption key $p k \in\{0,1\}^{\mathrm{DE} \cdot \mathrm{pkl}(\lambda)}$ and a secret decryption key $s k$. Given inputs $1^{\lambda}, \pi, p k$, a message $m \in\{0,1\}^{\mathrm{DE} \cdot \mathrm{ml}(\lambda)}$ and access to an oracle R: $\{0,1\}^{\text {DE.ROil }(\lambda)} \rightarrow\{0,1\}^{\text {DE.ROol }(\lambda)}$, the deterministic encryption algorithm DE. Enc outputs a ciphertext $c=\mathrm{DE} . \mathrm{Enc}^{\mathrm{R}}\left(1^{\lambda}, \pi, p k, m\right)$. Given inputs $1^{\lambda}, \pi, s k$, a ciphertext $c$ and oracle $\mathrm{R}$, the deterministic decryption algorithm DE.Dec output either a message $m \in\{0,1\}^{\text {DE.ml( }(\lambda)}$, or $\perp$. Here DE.pl, DE.pkl, DE.ml: $\mathbb{N} \rightarrow \mathbb{N}$ are the parameter, public key and message length functions of $D E$, respectively, while DE.ROil, DE.ROol: $\mathbb{N} \rightarrow \mathbb{N}$ are the RO input and output length functions, respectively. Correctness requires that for all $\lambda \in \mathbb{N}$, all $\pi \in\left[\operatorname{DE} \cdot \operatorname{Pg}\left(1^{\lambda}\right)\right]$, all $[(p k, s k) \in$ 


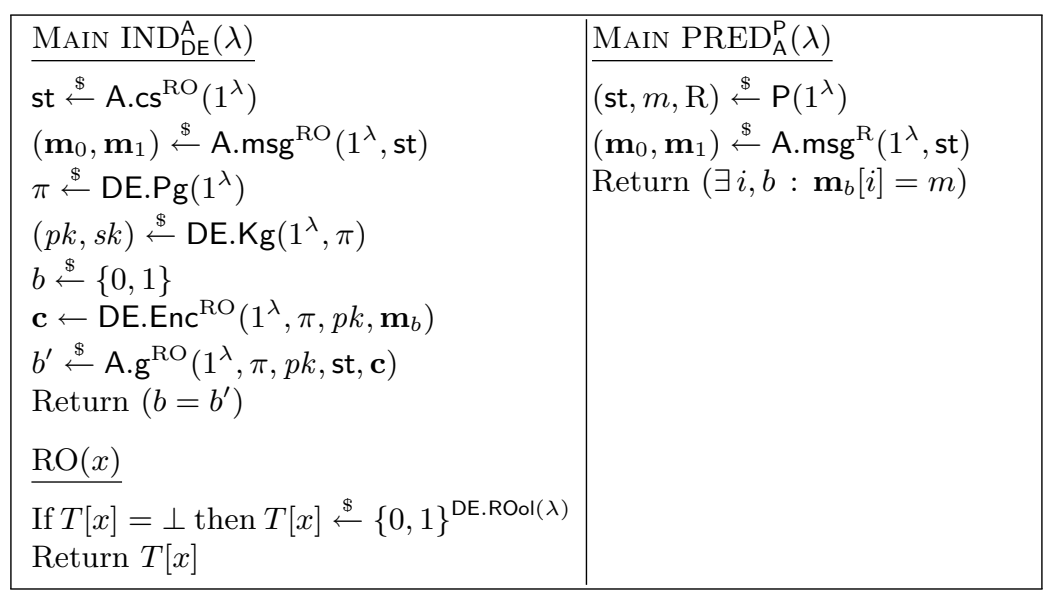

Fig. 2. The IND game used to define security of D-PKE scheme DE and the PRED game used to define unpredictability of adversary $\mathrm{A}$

[DE.Kg $\left.\left(1^{\lambda}, \pi\right)\right]$, all $m \in\{0,1\}^{\mathrm{DE} \cdot \mathrm{ml}(\lambda)}$ and all $\mathrm{R} \in \operatorname{Maps}[\operatorname{DE} \cdot \operatorname{ROil}(\lambda), \operatorname{DE} \cdot \operatorname{ROol}(\lambda)]$ we have DE.Dec ${ }^{\mathrm{R}}\left(1^{\lambda}, \pi, s k\right.$, DE.Enc $\left.{ }^{\mathrm{R}}\left(1^{\lambda}, \pi, p k, m\right)\right)=m$. We extend DE.Enc to take input vectors of messages by defining DE. Enc ${ }^{\mathrm{R}}\left(1^{\lambda}, \pi, p k, \mathbf{m}\right)=\left(\mathrm{DE}^{\mathrm{E}} \mathrm{Enc}^{\mathrm{R}}\left(1^{\lambda}\right.\right.$, $\left.\pi, p k, \mathbf{m}[1]), \ldots, \mathrm{DE} . \mathrm{Enc}^{\mathrm{R}}\left(1^{\lambda}, \pi, p k, \mathbf{m}[|\mathbf{m}|]\right)\right)$, and similarly we let DE.Dec ${ }^{\mathrm{R}}\left(1^{\lambda}\right.$, $\pi, s k, \mathbf{c})=\left(\mathrm{DE} . \operatorname{Dec}^{\mathrm{R}}\left(1^{\lambda}, \pi, s k, \mathbf{c}[1]\right), \ldots, \mathrm{DE} . \operatorname{Dec}^{\mathrm{R}}\left(1^{\lambda}, \pi, s k, \mathbf{c}[|\mathbf{c}|]\right)\right)$. We say that DE is a standard-model (SM) scheme if DE.Enc, DE.Dec make no oracle queries, and in this case we will omit the superscript $R$ to DE.Enc, DE.Dec.

IND SECURITY. We define IND security of a D-PKE scheme DE following BFOR [10]. An IND adversary A specifies a common-state generation algorithm A.cs, a message-generation algorithm A.msg and a guessing algorithm A.g, all PT. On input $1^{\lambda}$, algorithm A.cs generates state information st that will be passed to both A.msg and A.g. Algorithm A.msg, on input $1^{\lambda}$, st returns a pair $\left(\mathbf{m}_{0}, \mathbf{m}_{1}\right)$ of vectors of messages with $\left|\mathbf{m}_{0}\right|=\left|\mathbf{m}_{1}\right|=A \cdot n m(\lambda)$ and $\mathbf{m}_{0}[i], \mathbf{m}_{1}[i] \epsilon$ $\{0,1\}^{\mathrm{DE} \cdot \mathrm{ml}(\lambda)}$, where A.nm: $\mathbb{N} \rightarrow \mathbb{N}$ is the number-of-messages function associated to $A$. It is required that the strings (messages) $\mathbf{m}_{0}[1], \ldots, \mathbf{m}_{0}\left[\left|\mathbf{m}_{0}\right|\right]$ are distinct and the strings (messages) $\mathbf{m}_{1}[1], \ldots, \mathbf{m}_{1}\left[\left|\mathbf{m}_{1}\right|\right]$ are distinct. Also associated to DE are functions DE.ROil, DE.ROol, the input and output length of the RO that is used by the scheme. We say that $A$ is a standard-model adversary if it makes no oracle queries, and in this case we may omit giving it an oracle.

The $\operatorname{IND}_{\mathrm{DE}}^{\mathrm{A}}(\lambda)$ game associated with DE and adversary $\mathrm{A}$ is described on the left of Fig. 2. We define the advantage of $A$ via $\mathbf{A d v}_{\mathrm{DE}, \mathrm{A}}^{\mathrm{ind}}(\lambda)=2 \cdot \operatorname{Pr}\left[\operatorname{IND}_{\mathrm{DE}}^{\mathrm{A}}(\lambda)\right]-1$ for all $\lambda \in \mathbb{N}$. If $\mathcal{A}$ is a class (set) of adversaries then we say that $\operatorname{DE}$ is $\operatorname{IND}[\mathcal{A}]$ secure if $\operatorname{Adv}_{\mathrm{DE}, \mathrm{A}}^{\text {ind }}(\cdot)$ is negligible for all $\mathrm{A} \in \mathcal{A}$. It is convenient to view $\operatorname{IND}[\mathcal{A}]$ as a set, so that $\operatorname{DE} \in \operatorname{IND}[\mathcal{A}]$ iff $\operatorname{DE}$ is $\operatorname{IND}[\mathcal{A}]$-secure. With this framework, we can now obtain various variants of the notion by varying and restricting the class $\mathcal{A}$.

First, we must impose the necessary condition that messages being encrypted have high min-entropy. In game PRED of Fig. 2, the predictor adversary $\mathrm{P}$ begins 
by specifying st and a guess $m$ as to a message that A.msg will generate. It also specifies the function $\mathrm{R} \in \operatorname{Maps}[\operatorname{DE} . \operatorname{ROil}(\lambda), \operatorname{DE} . \operatorname{ROol}(\lambda)]$ that will play the role of the RO. This captures the requirement that high min-entropy is required across all choices of the oracle. We let $\operatorname{Adv}_{A, P}^{\text {pred }}(\lambda)=\operatorname{Pr}\left[\operatorname{PRED}_{A}^{P}(\lambda)\right]$ for all $\lambda \in \mathbb{N}$. We say that $A$ is unpredictable if $\mathbf{A} \mathbf{d v}_{A, P}^{\text {pred }}(\cdot)$ is negligible for all $P$. We stress that here $\mathrm{P}$ is not restricted to $\mathrm{PT}$ but may be computationally unbounded. If $\mathrm{A}$ is a standard model adversary then we may omit $R$ in the output of $P$.

Following [10], our adversaries $A$ are three stage. If A.cs always returns $\varepsilon$ then we say that $A$ has trivial initial state and we may refer to $A$ as a two-stage adversary. In BFOR [10], definitions of security are relative to two-stage adversaries, three-stage ones being introduced in order to facilitate proofs. Accordingly, our definitions of security will also be in terms of two-stage adversaries.

We are now ready to define adversary classes of interest. We consider two dimensions: the model (ROM or SM), and the type of computation (non-uniform or uniform). With two choices in each category, we get 4 classes of adversaries and 4 corresponding notions of security for D-PKE. Proceeding to the details, we let $\mathcal{A}_{3}$ be the class of all PT, 3-stage, unpredictable adversaries and $\mathcal{A}_{2} \subseteq \mathcal{A}_{3}$ the class of all PT, 2-stage unpredictable adversaries. We let $\mathcal{A}^{\text {rom }}$ denote the class of ROM adversaries, and $\mathcal{A}^{\mathrm{sm}} \subseteq \mathcal{A}^{\text {rom }}$ the class of SM adversaries. We let $\mathcal{A}^{\text {nu }}$ denote the class of non-uniform adversaries, and $\mathcal{A}^{\mathrm{u}} \subseteq \mathcal{A}^{\text {nu }}$ the class of uniform adversaries. Then our 4 classes are $\mathcal{A}_{2}^{\mathrm{xm}-\mathrm{xu}}=\mathcal{A}^{\mathrm{xm}} \cap \mathcal{A}^{\mathrm{xu}} \cap \mathcal{A}_{2}$ for $\mathrm{xm} \in\{\mathrm{rom}, \mathrm{sm}\}$ and $\mathrm{xu} \in\{\mathrm{nu}, \mathrm{u}\}$. The 4 corresponding notions of D-PKE security are $\operatorname{IND}\left[\mathcal{A}_{2}^{\mathrm{xm}-\mathrm{xu}}\right]$ for $\mathrm{xm} \in\{$ rom, sm $\}$ and $\mathrm{xu} \in\{\mathrm{nu}, \mathrm{u}\}$.

\section{Does SM Security Imply ROM Security?}

We now explore if a D-PKE scheme that is IND-secure in the standard model $(\mathrm{SM})$ is IND-secure in the ROM.

Problem AND APPROACH. It is easy to show that a SM R-PKE scheme retains its security in the ROM, where the adversary has access to the random oracle, because a SM adversary can simply simulate the random oracle for a ROM adversary. Indeed, that SM security implies ROM security seems to have been viewed as trivial and true for any primitive. We are about to see, however, that for D-PKE the answer is less clear.

We are given a SM D-PKE scheme DE that is secure in the SM, meaning its algorithms make no calls to RO and it is secure against adversaries that make no calls to RO. We ask if DE remains secure in the ROM, meaning when the adversary is allowed to query RO. The reason an adversary A may be able now to do more is that A.msg may create messages that depend in some clever way on RO and then A.g could exploit the fact that it has access to the same RO to figure out something about the messages from the ciphertexts. Intuitively, however, it is difficult to see how this could happen because messages are required to have high min-entropy even given RO. However, it is not clear how to prove it, which raises the question of whether it is even true. The difficulty is that no 
communication is allowed from the message-finding stage of the adversary to the guessing stage, and so a simulating SM adversary has no obvious way to ensure that these two stages have a common view of the random oracle it is simulating.

We will first present Lemma 1 showing the claim is true in the 3-stage adversary formulation of the IND games. Namely given a SM D-PKE scheme and given a 3-stage ROM adversary $A$, we show how to simulate $A$ with a 3 -stage SM adversary $B$ so that the latter has the same advantage as $A$. The proof uses a $q(\cdot)$-wise independent hash function, with the polynomial $q$ depending on A, as the common initial state created by B.cs. The lemma is true both in the uniform and the non-uniform settings. However, recall that IND security is defined with respect to adversaries that have trivial initial state, meaning are two stage. And in our reduction, B will have non-trivial initial state even if A has trivial initial state. So the lemma does not directly show that IND in the SM implies IND in the ROM. In the non-uniform case, however, we can flatten the constructed 3-stage adversary $\mathrm{B}$ into an equivalent one with trivial initial state, thereby concluding that if SM D-PKE scheme DE is in $\operatorname{IND}\left[\mathcal{A}_{2}^{\mathrm{sm}-\mathrm{nu}}\right]$ then it is also in $\operatorname{IND}\left[\mathcal{A}_{2}^{\text {rom-nu }}\right]$. In the uniform setting we have no obvious way to remove the non-trivial initial state of $\mathrm{B}$, and thus are not able to conclude that $\mathrm{DE}$ being in $\operatorname{IND}\left[\mathcal{A}_{2}^{\mathrm{sm}-\mathrm{u}}\right]$ implies it is in $\operatorname{IND}\left[\mathcal{A}_{2}^{\mathrm{rom}-\mathrm{u}}\right]$. This very basic question (surprisingly) remains open.

$q$-WISE INDEPENDENT FUNCTION FAMILIES. We say that a family HF of functions is $q(\cdot)$-wise independent if for all $\lambda \in \mathbb{N}$, all $q(\lambda)$-vectors $\mathbf{x}$ over $\{0,1\}^{\mathrm{HF} . \mathrm{il}(\lambda)}$ all of whose entries are distinct, and all $q(\lambda)$-vectors $\mathbf{y}$ over $\{0,1\}^{\mathrm{HF} . \mathrm{ol}(\lambda)}$ we have $\operatorname{Pr}\left[\operatorname{HF}\left(1^{\lambda}, k, \mathbf{x}\right)=\mathbf{y}\right]=2^{-q(\lambda) \cdot \mathrm{HF} . \mathrm{ol}(\lambda)}$, where the probability is over $k$ chosen at random from $\{0,1\}^{\mathrm{HF} . \mathrm{kl}(\lambda)}$.

From SM SECURITy TO ROM SECURITy With 3 STAGES. The following lemma says that for any SM D-PKE scheme (meaning, the scheme algorithms do not call the RO), a 3-stage ROM adversary $A$ may be simulated by a 3-stage SM adversary $B$ who achieves the same advantage as $A$. It does not follow that a 2-stage ROM adversary can be simulated by a 2-stage SM adversary since our constructed adversary B will have non-trivial initial state even if the given adversary $A$ had trivial initial state.

Lemma 1. Let DE be a standard-model D-PKE scheme. Let $\mathrm{A} \in \mathcal{A}^{\mathrm{rom}} \cap \mathcal{A}_{3}$ be a 3-stage, PT ROM adversary. Then there is a 3-stage, PT standard-model adversary $\mathrm{B} \in \mathcal{A}^{\mathrm{sm}} \cap \mathcal{A}_{3}$ such that

$$
\operatorname{Adv}_{\mathrm{DE}, \mathrm{B}}^{\text {ind }}(\lambda)=\mathbf{A d v}_{\mathrm{DE}, \mathrm{A}}^{\text {ind }}(\lambda)
$$

for all $\lambda \in \mathbb{N}$. Furthermore, if $\mathrm{A}$ is unpredictable then so is $\mathrm{B}$ and if $\mathrm{A}$ is uniform then so is $\mathrm{B}$.

Proof (Lemma 1). Without loss of generality, we assume that there exists a polynomial $q: \mathbb{N} \rightarrow \mathbb{N}$ such that for all $\lambda \in \mathbb{N}$, adversary A always makes exactly $q(\lambda) \mathrm{RO}$ queries in game $\operatorname{IND}_{\mathrm{DE}}^{\mathrm{A}}(\lambda)$. Let $\mathrm{HF}$ be a $q(\cdot)$-wise independent family of functions with HF.il = DE.ROil and HF.ol = DE.ROol. We define SM adversary $\mathrm{B}$ as follows: 


\begin{tabular}{|c|c|}
\hline B. $\operatorname{cs}\left(1^{\lambda}\right)$ & B. $m s g\left(1^{\lambda}, s t_{B}\right)$ \\
\hline $\begin{array}{l}k \stackrel{\$}{\leftarrow}\{0,1\}^{\mathrm{HF} \cdot \mathrm{kl}(\lambda)} \\
\mathrm{st}_{\mathrm{A}} \stackrel{\$}{\leftarrow} \mathrm{A} \cdot \mathrm{cs}^{\mathrm{ROSim}}\left(1^{\lambda}\right) \\
\text { Return }\left(k, \mathrm{st}_{\mathrm{A}}\right)\end{array}$ & $\begin{array}{l}\overline{\left(k, \mathrm{st}_{\mathrm{A}}\right) \leftarrow \mathrm{st}_{\mathrm{B}}} \\
\left(\mathbf{m}_{0}, \mathbf{m}_{1}\right) \stackrel{\$}{\leftarrow} \mathrm{A} \cdot \mathrm{msg}^{\mathrm{ROSim}}\left(1^{\lambda}, \mathrm{st}_{\mathrm{A}}\right) \\
\operatorname{Return}\left(\mathbf{m}_{0}, \mathbf{m}_{1}\right)\end{array}$ \\
\hline $\operatorname{ROSim}(x)$ & $\underline{\operatorname{ROSim}(x)}$ \\
\hline$\overline{\operatorname{Return~} \mathrm{HF}}\left(1^{\lambda}, k, x\right)$ & Return $\operatorname{HF}\left(1^{\lambda}, k, x\right)$ \\
\hline
\end{tabular}

$$
\begin{aligned}
& \frac{\mathrm{B} \cdot \mathrm{g}\left(1^{\lambda}, \pi, p k, \mathrm{st}_{\mathrm{B}}, \mathbf{c}\right)}{\left(k, \mathrm{st}_{\mathrm{A}}\right) \leftarrow \mathrm{st}_{\mathrm{B}}} \\
& b^{\prime} \stackrel{\$}{\leftarrow} \mathrm{A} \cdot \mathrm{g}^{\mathrm{ROSim}}\left(1^{\lambda}, \pi, p k, \mathrm{st}_{\mathrm{A}}, \mathbf{c}\right)
\end{aligned}
$$

Return $b^{\prime}$

\section{$\operatorname{ROSim}(x)$}

Return $\operatorname{HF}\left(1^{\lambda}, k, x\right)$

That is, B.cs picks at random a key defining a member of HF and passes it to B.msg, B.g. The latter use the function $\operatorname{HF}\left(1^{\lambda}, k, \cdot\right)$ to simulate the $\mathrm{RO}$ of A, via the ROSim procedure. Since A makes at most $q(\lambda)$ queries to RO, the $q(\lambda)$-wise independence of the family should result in a perfect simulation of the RO. Also, since both B.msg and B.g use the same function, A.msg and A.g will see a consistent RO across their two stages. As a result we expect that (1) is true.

Formally proving that (1) is true, however, is not straightforward because the $\mathrm{RO}$ queries are adaptive and $q(\cdot)$-wise independence is a non-adaptive condition, so some care must be taken. In [8] we provide an analysis that handles this, and do not discuss it further here.

It is clear that if $A$ is uniform then so is B. Assuming $A$ is unpredictable we now have to show that $B$ is unpredictable. Let $P_{B}$ be a predictor adversary for $B$. We define a predictor adversary $P_{A}$ for $A$ as follows. On input $1^{\lambda}$ it runs $\mathrm{P}_{\mathrm{B}}\left(1^{\lambda}\right)$ to get back $\left(\mathrm{st}_{\mathrm{B}}, m\right)$. (Since $\mathrm{B}$ is $\mathrm{SM}, \mathrm{P}_{\mathrm{B}}$ returns a pair, not a triple.) It parses $\mathrm{st}_{\mathrm{B}}$ as $\left(k, \mathrm{st}_{\mathrm{A}}\right)$ and returns $\left(\mathrm{st}_{\mathrm{A}}, m, \operatorname{HF}\left(1^{\lambda}, k, \cdot\right)\right)$. Then we have $\mathbf{A d v}_{\mathrm{A}, \mathrm{P}_{\mathrm{A}}}^{\text {pred }}(\cdot)=\mathbf{A} \mathbf{d} \mathbf{v}_{\mathrm{B}, \mathrm{P}_{\mathrm{B}}}^{\text {pred }}(\cdot)$. But the LHS is negligible by assumption, so the RHS is negligible as well.

We note that alternatively, in place of a family of $q(\cdot)$-wise independent functions, we could have used a PRF, the key being chosen by B.cs and included in the state so that it is passed to B.msg, B.g. The latter would use the PRF under this key to simulate the RO for A.msg, A.g, respectively. O'Neill used this technique [35, Lemma 3.3.2] to partially remove the $\mathrm{RO}$ for a restricted class of D-PKE schemes.

SM SECURITY IMPLIES ROM SECURITY IN THE NON-UNIFORM SETTING. The following theorem uses Lemma 1 to show that if a D-PKE scheme DE is INDsecure in the standard model with respect to non-uniform adversaries, then it is IND-secure in the ROM with respect to non-uniform adversaries. The proof uses non-uniformity in a crucial way, and hence cannot be adapted to the uniform setting. 
Theorem 1. Let $\mathrm{DE}$ be a SM D-PKE scheme such that $\mathrm{DE} \in \operatorname{IND}\left[\mathcal{A}_{2}^{\mathrm{sm}-\mathrm{nu}}\right]$. Then $\operatorname{DE} \in \operatorname{IND}\left[\mathcal{A}_{2}^{\mathrm{rom}-\mathrm{nu}}\right]$.

Proof. Let $\mathrm{A} \in \mathcal{A}_{2}^{\text {rom-nu }}$ be an unpredictable, non-uniform PT ROM adversary with trivial initial state. By Lemma 1 , we get an unpredictable, non-uniform PT SM adversary $\mathrm{B} \in \mathcal{A}^{\mathrm{sm}} \cap \mathcal{A}^{\mathrm{nu}} \cap \mathcal{A}_{3}$ such that $\operatorname{Adv}_{\mathrm{DE}, \mathrm{B}}^{\text {ind }}(\cdot)=\operatorname{Adv}_{\mathrm{DE}, \mathrm{A}}^{\text {ind }}(\cdot)$. However, B.cs is not trivial, so the assumption that $\operatorname{DE} \in \operatorname{IND}\left[\mathcal{A}_{2}^{\mathrm{sm}-\mathrm{nu}}\right]$ does not allow us to conclude that $\operatorname{Adv}_{\mathrm{DE}, \mathrm{B}}^{\text {ind }}(\cdot)$, and hence $\operatorname{Adv}_{\mathrm{DE}, \mathrm{A}}(\cdot)$, is negligible. We modify $B$ to an unpredictable, trivial initial state, non-uniform SM adversary $\mathrm{C} \in \mathcal{A}_{2}^{\mathrm{sm}-\mathrm{nu}}$ with $\operatorname{Adv}_{\mathrm{DE}, \mathrm{C}}^{\text {ind }}(\cdot)=\mathbf{A d v}_{\mathrm{DE}, \mathrm{B}}^{\text {ind }}(\cdot)$. Now the assumption that $\mathrm{DE} \in$ $\operatorname{IND}\left[\mathcal{A}_{2}^{\text {sm-nu }}\right]$ means that $\operatorname{Adv}_{\mathrm{DE}, \mathrm{C}}^{\text {ind }}(\cdot)$ is negligible and hence so is $\operatorname{Adv}_{\mathrm{DE}, \mathrm{A}}^{\text {ind }}(\cdot)$, showing that $\mathrm{DE} \in \operatorname{IND}\left[\mathcal{A}_{2}^{\text {rom-nu}}\right]$ as desired. To obtain $\mathrm{C}$ from $\mathrm{B}$, we simply use coin fixing, namely we hardwire a best choice of the key $k$ chosen randomly by B.cs $\left(1^{\lambda}\right)$ into the circuits C.msg $\left(1^{\lambda}, \cdots\right)$ and C.g $\left(1^{\lambda}, \cdots\right)$ while letting C.cs always return $\varepsilon$.

We note that the issues and difficulties associated with showing that SM security implies ROM security could also be viewed as arising from definitional shortcomings of existing formulations, and addressed definitionally, for example by making the three-stage definition the basic one with respect to which security is measured. Lemma 1 directly implies that if DE is a SM D-PKE scheme, then: (1) If $\operatorname{DE} \in \operatorname{IND}\left[\mathcal{A}^{\mathrm{sm}} \cap \mathcal{A}^{\mathrm{u}} \cap \mathcal{A}_{3}\right]$ then $\operatorname{DE} \in \operatorname{IND}\left[\mathcal{A}^{\mathrm{rom}} \cap \mathcal{A}^{\mathrm{u}} \cap \mathcal{A}_{3}\right]$ and (2) If $\mathrm{DE} \in \operatorname{IND}\left[\mathcal{A}^{\mathrm{sm}} \cap \mathcal{A}^{\mathrm{nu}} \cap \mathcal{A}_{3}\right]$ then $\mathrm{DE} \in \operatorname{IND}\left[\mathcal{A}^{\mathrm{rom}} \cap \mathcal{A}^{\mathrm{nu}} \cap \mathcal{A}_{3}\right]$. That is, for 3stage adversaries, SM security implies ROM security both in the uniform and non-uniform settings. However the question of whether the implication holds for two-stage adversaries and the current definitions would still be interesting.

\section{$5 \quad$ Is SOA Security Achievable?}

We initiate an investigation of SOA security for D-PKE. We provide definitions and then show that the goal is impossible to achieve in the SM, meaning no SM D-PKE scheme achieves it.

What makes this interesting is that the difficulty of achieving SOA security in the R-PKE case arises from the fact that an attacker obtains not only messages but the coins underlying the opened ciphertexts. If it only obtained messages, security is easy to achieve [17]. Since in D-PKE there are no coins, one might think security would be also easy to achieve. But in fact this is not true.

Preliminaries. We let $\perp_{n}$ denote the vector of length $n$ all of whose entries are $\perp$. For a set $I \subseteq[|\mathbf{x}|]$ we let $\mathbf{x}[I]$ denote the $|\mathbf{x}|$-vector whose $i$-th component is $\mathbf{x}[i]$ if $i \in I$ and $\perp$ otherwise.

Collision resistance of a function family $\mathrm{HF}$ is defined via game $\mathrm{CR}_{\mathrm{HF}}^{\mathrm{X}}(\lambda)$ associated to HF, adversary $\mathrm{X}$ and $\lambda \in \mathbb{N}$. The game starts by picking $k \stackrel{\&}{\leftarrow}$ $\{0,1\}^{\mathrm{HF} . \mathrm{kl}(\lambda)}$. Then $\mathrm{X}$ is run with inputs $1^{\lambda}, k$ to return $x_{0}, x_{1} \in\{0,1\}^{\mathrm{HF} . \mathrm{il}(\lambda)}$. The game returns true if $x_{0} \neq x_{1}$ and $\operatorname{HF}\left(1^{\lambda}, k, x_{0}\right)=\operatorname{HF}\left(1^{\lambda}, k, x_{1}\right)$, and false 


\begin{tabular}{|c|c|c|}
\hline MAIN REAL ${ }_{\mathrm{DE}}^{\mathrm{A}}(\lambda)$ & MAIN IDEAL $\operatorname{IDE}_{\mathrm{DE}}^{\mathrm{A}, \mathrm{S}}(\lambda)$ & MAIN PRED $\operatorname{PA}_{A}^{P}(\lambda)$ \\
\hline$k \stackrel{\$}{\longleftarrow} \mathrm{A} \cdot \operatorname{cs}\left(1^{\lambda}\right)$ & $k \stackrel{\$}{\leftarrow} \mathrm{A} \cdot \operatorname{cs}\left(1^{\lambda}\right)$ & $(I$, st $) \stackrel{\$}{\longleftarrow} \mathrm{P}\left(1^{\lambda}\right)$ \\
\hline $\mathbf{m} \stackrel{\$}{\leftarrow} \operatorname{A} \cdot \mathrm{msg}\left(1^{\lambda}\right)$ & $\mathbf{m} \stackrel{\$}{\leftarrow}$ A.msg $\left(1^{\lambda}\right)$ & $\mathbf{m} \stackrel{\$}{\leftarrow} \mathrm{A} \cdot \mathrm{msg}\left(1^{\lambda}\right)$ \\
\hline$\pi \stackrel{\$}{\leftarrow}$ DE.Pg $\left(1^{\lambda}\right)$ & st $\stackrel{\$}{\longleftarrow} \mathrm{S}^{\mathrm{COR}}\left(1^{\lambda}, k\right)$ & $m \stackrel{\$}{\leftarrow} \mathrm{P}(\mathrm{st}, \mathbf{m}[I])$ \\
\hline$(p k, s k) \stackrel{\$}{\longleftarrow} \mathrm{DE} \cdot \mathrm{Kg}\left(1^{\lambda}, \pi\right)$ & $w \stackrel{\$}{\leftarrow} \mathrm{A} \cdot \mathrm{g}\left(1^{\lambda}, k, I, \mathrm{st}, \mathbf{m}[I]\right)$ & Return $(\exists i \notin I: \mathbf{m}[i]=$ \\
\hline $\mathbf{c} \stackrel{\$}{\leftarrow} \operatorname{DE} . \operatorname{Enc}\left(1^{\lambda}, \pi, p k, \mathbf{m}\right)$ & $\operatorname{Return}\left(w=\operatorname{A} \cdot \mathbf{f}\left(1^{\lambda}, \mathbf{m}\right)\right)$ & \\
\hline st $\stackrel{\$}{\longleftarrow}$ & $\operatorname{CoR}(I)$ & \\
\hline A. $\operatorname{cor}_{\$}^{\mathrm{CoR}}\left(1^{\lambda}, \pi, p k, k, \mathbf{c}\right)$ & $\overline{\text { Return } \mathbf{m}[I]}$ & \\
\hline$w \stackrel{\$}{\leftarrow} \mathrm{A} \cdot \mathrm{g}\left(1^{\lambda}, k, I, \mathbf{s t}, \mathbf{m}[I]\right)$ & & \\
\hline $\operatorname{Return}\left(w=\operatorname{A} \cdot \mathbf{f}\left(1^{\lambda}, \mathbf{m}\right)\right)$ & & \\
\hline $\operatorname{CoR}(I)$ & & \\
\hline$\overline{\text { Return } \mathbf{m}[I]}$ & & \\
\hline
\end{tabular}

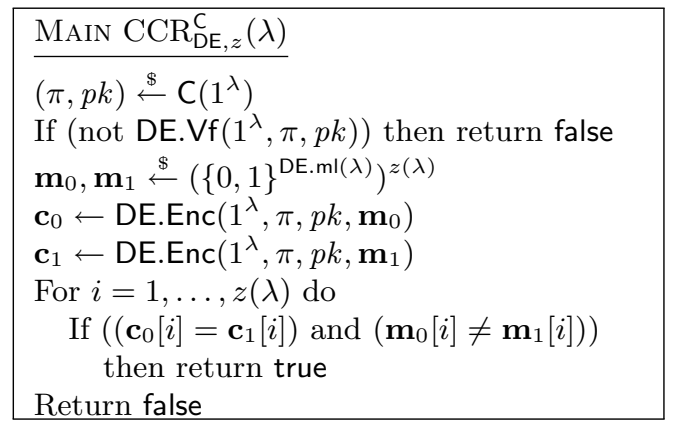

Fig. 3. The REAL, IDEAL, PRED and CCR games

otherwise. The advantage of $\mathbf{X}$ is defined as $\mathbf{A d v}_{\mathrm{HF}, \mathrm{X}}^{\mathrm{cr}}(\lambda)=\operatorname{Pr}\left[\mathrm{CR}_{\mathrm{HF}}^{\mathrm{X}}(\lambda)\right]$ and we say that HF is collision resistant if $\operatorname{Addv}_{\mathrm{HF}, \mathrm{X}}^{\mathrm{cr}}(\cdot)$ is negligible for all PT $\mathrm{X}$.

DEFINING SOA SECURITy. Providing a meaningful definition of SOA-security for D-PKE takes some care. A definition based on semantic security for relations, as given for R-PKE in $[9,12]$, is trivially unachievable for D-PKE because a ciphertext is already partial information about a plaintext. Thus we consider semantic security for functions, where the adversary, given ciphertexsts, aims to figure out a function of the message, this function not being given the public key and thus unable to encrypt. Additionally we must continue to require that messages do not depend on the public key and are unpredictable. Our definition is simulation-based and combines ideas from the basic (non-SOA) definitions of secure D-PKE $[5,10]$ with ideas from the definitions of SOA-security for R-PKE from $[9,12]$.

In Fig. 3 is the "real" game REAL $\mathrm{DE}_{\mathrm{DE}}^{\mathrm{A}}$ associated to D-PKE scheme DE and adversary A. PT common state generation algorithm A.cs is executed on input $1^{\lambda}$ to get a common state $k$ that will be passed to the A.cor stage of A. (Other stages can get it too, but since our results are negative, not giving it only makes the results stronger.) Then PT message generator A.msg is executed on input 
$1^{\lambda}$ to get a $A \cdot n m(\lambda)$-vector of messages over $\{0,1\}^{\mathrm{DE} \cdot \mathrm{ml}(\lambda)}$, where A.nm is the number-of-messages function associated to $A$. Then public parameters and keys are generated. (It is important that the messages do not depend on the public parameters or public key of DE for the same reason as with PRIV [5] and IND [10], namely that otherwise security is trivially unachievable.) Then the vector of messages is encrypted, component-wise, to get a vector $\mathbf{c}$ of ciphertexts. The PT corruption algorithm A.cor gets $1^{\lambda}, \pi, p k, k, \mathbf{c}$ and an oracle CoR to which it is allowed exactly one query, this consisting of a subset $I$ of $[A \cdot n m(\lambda)]$, indicating positions at which it wants $\mathbf{m}$ opened. In response it gets $\mathbf{m}[I]$, meaning the values $\mathbf{m}[i]$ for $i \in I$, and returns state information st. The PT guessing algorithm A.g gets $1^{\lambda}, k, I, \mathrm{st}, \mathbf{m}[I]$, where $I$ is the CoR-query previously made by A.cor and recorded by the game, and outputs a guess $w$ as to the value of A.f $\left(1^{\lambda}, \mathbf{m}\right)$. Here deterministic PT algorithm A.f, called the information function, represents the information about $\mathbf{m}$ that the adversary is trying to compute. The game returns true iff the guess is correct.

The "ideal" game IDEAL $L_{D E}^{A, S}$ of Fig. 3 is associated to DE, adversary $A$ and a simulator $\mathrm{S}$. Here, the common state and message vector are chosen as before, but the game neither chooses parameters and public key, nor generates any ciphertexts. The simulator is given no information about $\mathbf{m}$, but has access to oracle Cor, to which it is allowed exactly one query, this consisting of a subset $I$ of $[A \cdot n m(\lambda)]$. In response $\mathbf{S}$ gets $\mathbf{m}[I]$ and must then return state information st that should resemble the output of A.cor. The rest is as in the real game.

We need to restrict A.msg to reflect the inherent weaknesses of D-PKE, analogous to the restrictions made in defining PRIV and IND. Namely we require a message-distinctness condition and a message unpredictability (high min-entropy) condition. Before detailing definitions, we note that the A.msg in Theorem 2 simply outputs uniform, independently distributed messages of superlogarithmic length, so both the conditions will be trivially met, and thus a reader can skip the rest of this paragraph if they wish. Proceeding, since ciphertext equality leaks plaintext equality in D-PKE, we require the following messagedistinctness condition: there is a negligible function $\nu$ such that $\operatorname{Pr}[\exists i, j:(i \neq$ $j) \wedge(\mathbf{m}[i]=\mathbf{m}[j])] \leq \nu(\lambda)$ where the probability is over $\mathbf{m} \stackrel{\&}{\leftarrow} \mathrm{A} \cdot \mathbf{m s g}\left(1^{\lambda}\right)$. Second, we require that $\mathbf{A}$ is unpredictable, which we define to mean that $\mathbf{A d v}_{\mathrm{A}, \mathrm{P}}^{\text {pred }}(\lambda)=$ $\operatorname{Pr}\left[\operatorname{PRED}_{A}^{P}(\lambda)\right]$ is negligible for all $P$ (we emphasize that here $P$ is not restricted to be PT), where game PRED ${ }_{A}^{P}$ is shown on the middle, bottom of Fig. 3. The unpredictability condition we define here is very strong, requiring that each component message of $\mathbf{m}$ has high min-entropy even given the others, but this only strengthens our results since they are negative. We let $\mathcal{A}^{\text {soa }}$ denote the class of all PT A that satisfy the message distinctness and unpredictability conditions.

We define the soa-advantage of an adversary $A$ with respect to DE and a simulator S via

$$
\operatorname{Adv}_{\mathrm{DE}, \mathrm{A}, \mathrm{S}}^{\text {soa }}(\lambda)=\operatorname{Pr}\left[\operatorname{REAL}_{\mathrm{DE}}^{\mathrm{A}}(\lambda)\right]-\operatorname{Pr}\left[\operatorname{IDEAL}_{\mathrm{DE}}^{\mathrm{A}, \mathrm{S}}(\lambda)\right]
$$

for all $\lambda \in \mathbb{N}$. We say that $\mathrm{DE}$ is $\mathrm{SOA}$-secure if for all $\mathrm{A} \in \mathcal{A}^{\text {soa }}$, there exists a PT simulator $\mathbf{S}$ such that $\mathbf{A d v}_{\mathrm{DE}, \mathrm{A}, \mathrm{S}}^{\text {soa }}(\cdot)$ is negligible. 
The definitions and results here are all in the standard model. Our impossibility result does not rule out achieving an appropriate (programmable) ROM version of our definition of SOA-security for D-PKE. In [8] we further discuss the definitional choices made here.

APPROACH. BDWY [9] show that if CR hash functions exist then any R-PKE scheme satisfying a certain binding property they define is not SOA-secure. Roughly, binding says that encryption remains injective even on dishonestlychosen public keys. Not all R-PKE schemes satisfy this binding property, but many common ones do, and the BDWY result shows in particular that IND-CPA does not imply SOA for R-PKE. In the D-PKE case, rather than ask for schemes that are binding, we introduce a verification algorithm that, given a dishonestlygenerated public key, tests the extent to which the encryption induced by this key is an injective function. If it is far from injective, verification will catch it, and otherwise we have some sort of binding. We then show that such a verification algorithm exists for every D-PKE scheme. Adapting the technique of BDWY we can then use this to show that no D-PKE scheme is SOA-secure.

INJECTIVITY VERIFICATION. Let DE be a D-PKE scheme. A verification algorithm DE.Vf for DE is a PT algorithm that takes as input $1^{\lambda}, \pi, p k$ and returns a boolean value. Here, $\pi$ and $p k$ play the role of parameters and a public key but are to be thought of as adversarially chosen and not necessarily ones that would actually arise in honest parameter and key generation. Informally, $\mathrm{DE} . \mathrm{Vf}$ checks if the provided $\pi, p k$ induce an almost injective function on valid DE messages. We impose a requirement we call completeness, which says that for all $\lambda \in \mathbb{N}$, all $\pi \in\left[\operatorname{DE} . \operatorname{Pg}\left(1^{\lambda}\right)\right]$ and all $(p k, s k) \in\left[\operatorname{DE} . \operatorname{Kg}\left(1^{\lambda}, \pi\right)\right]$ we have $\mathrm{DE} . \mathrm{Vf}\left(1^{\lambda}, \pi, p k\right)=$ true. That is, if the parameters and key are honestly chosen then the verifier accepts. To formalize the requirement for adversarially chosen $\pi, p k$, consider the game described in Fig. 3, and define the ciphertext collision resistance advantage of an adversary $\mathrm{C}$ via $\mathbf{A d v}_{\mathrm{DE}, z, \mathrm{C}}^{\mathrm{ccr}}(\lambda)=\operatorname{Pr}\left[\mathrm{CCR}_{\mathrm{DE}, z}^{\mathrm{C}}(\lambda)\right]$. Here adversary $\mathrm{C}$ picks $\pi, p k$, so the encryption function induced by them, unlike that induced by an honestly-generated $\pi, p k$, may not be injective. The advantage of the adversary is the probability that it can get some non-injectivity to surface via collisions. The following lemma says that it is possible to design a verification algorithm that makes it hard for any adversary to defeat CCR.

Lemma 2. Let $\mathrm{DE}$ be a D-PKE scheme and $z: \mathbb{N} \rightarrow \mathbb{N}$. Define the verification algorithm DE.Vf as follows:

$\underline{\mathrm{DE} . \mathrm{Vf}\left(1^{\lambda}, \pi, p k\right)}$

If $(|\pi| \neq \mathrm{DE} \cdot \mathrm{pl}(\lambda)$ or $|p k| \neq \mathrm{DE} \cdot \operatorname{pkl}(\lambda))$ then return false

For $t=1, \ldots, z(\lambda)$ do

$\mathbf{m}_{0}^{\prime}[t] \stackrel{\&}{\leftarrow}\{0,1\}^{\mathrm{DE} \cdot \mathrm{ml}(\lambda)} ; \mathbf{m}_{1}^{\prime}[t] \stackrel{\&}{\leftarrow}\{0,1\}^{\mathrm{DE} \cdot \mathrm{ml}(\lambda)}$

If $\left(\left(\operatorname{DE} . \operatorname{Enc}\left(1^{\lambda}, \pi, p k, \mathbf{m}_{0}^{\prime}[t]\right)=\operatorname{DE} \cdot \operatorname{Enc}\left(1^{\lambda}, \pi, p k, \mathbf{m}_{1}^{\prime}[t]\right)\right) \wedge\left(\mathbf{m}_{0}^{\prime}[t] \neq \mathbf{m}_{1}^{\prime}[t]\right)\right)$

then return false

Return true 
Then DE.Vf is PT and complete. Also for any (not necessarily PT) adversary $\mathrm{C}$ we have $\mathbf{A d v}_{\mathrm{DE}, z, \mathrm{C}}^{\mathrm{ccr}}(\lambda) \leq \frac{1}{4}$ for all $\lambda \in \mathbb{N}$.

Proof. Lemma 2] For any $\lambda \in \mathbb{N}$, any $\pi \in\{0,1\}^{\mathrm{DE} \cdot \mathrm{pl}(\lambda)}$ and any $p k \in\{0,1\}^{\mathrm{DE} \cdot \mathrm{pkl}(\lambda)}$ let $\mathbf{C P}_{\mathrm{DE}}\left(1^{\lambda}, \pi, p k\right)$ equal the probability that there exists $t \in[z(\lambda)]$ such that

$$
\operatorname{DE} . \operatorname{Enc}\left(1^{\lambda}, \pi, p k, \mathbf{m}_{0}[t]\right)=\operatorname{DE} . \operatorname{Enc}\left(1^{\lambda}, \pi, p k, \mathbf{m}_{1}[t]\right) \text { and } \mathbf{m}_{0}[t] \neq \mathbf{m}_{1}[t]
$$

where the probability is over $\mathbf{m}_{0}, \mathbf{m}_{1} \stackrel{\&}{\leftarrow}\left(\{0,1\}^{\mathrm{DE} \cdot \mathrm{ml}(\lambda)}\right)^{z(\lambda)}$. In game CCR the probability that the test performed using DE.Vf is passed is $1-\mathbf{C P}_{\mathrm{DE}}\left(1^{\lambda}, \pi, p k\right)$. If such test is passed, the probability that some ciphertext collision appears (thus making the game CCR return true) is upper bounded by $\mathbf{C P}_{\mathrm{DE}}\left(1^{\lambda}, \pi, p k\right)$. Since passing the verification algorithm's test and having some ciphertext collision is the only combination in which game CCR returns true, for any adversary C, we get

$$
\begin{aligned}
& \mathbf{A d v}_{\mathrm{DE}, z, \mathrm{C}}^{\mathrm{ccr}}(\lambda) \\
& \leq \max _{\pi \in\{0,1\}^{\mathrm{DE} . \mathrm{pl}(\lambda)}} \max _{p k \in\{0,1\}^{\mathrm{DE} . \mathrm{pk}(\lambda)}}\left(\left(1-\mathbf{C P}_{\mathrm{DE}}\left(1^{\lambda}, \pi, p k\right)\right) \mathbf{C P}_{\mathrm{DE}}\left(1^{\lambda}, \pi, p k\right)\right) \\
& \leq \frac{1}{4}
\end{aligned}
$$

where the last inequality is from the maximum of the quadratic function.

IMPOSSIBILITY OF SOA SECURITY. In order to prove that a given D-PKE scheme $\mathrm{DE}$ is not SOA-secure we need to prove the existence of an adversary $\mathrm{A} \in \mathcal{A}^{\text {soa }}$ such that for every PT simulator $\mathrm{S}$, the function $\mathbf{A d v}_{\mathrm{DE}, \mathrm{A}, \mathrm{S}}^{\text {soa }}(\cdot)$ is not negligible. We assume a collision-resistant hash function HF in the following.

Theorem 2. Let $\mathrm{DE}$ be a D-PKE scheme such that $2^{-\mathrm{DE} . \mathrm{ml}(\cdot)}$ is negligible. Assume the existence of a collision-resistant family of functions. Then, there exists a PT adversary $\mathrm{A} \in \mathcal{A}^{\text {soa }}$ such that, for all PT simulators $\mathrm{S}$ there exists a function $\nu$ that is not negligible and is such that $\mathbf{A d v}_{\mathrm{DE}, \mathrm{A}, \mathrm{S}}^{\mathrm{soa}}(\lambda) \geq \nu(\lambda)$ for all $\lambda \in \mathbb{N}$. Furthermore, message sampler A.msg returns a vector of uniformly and independently distributed messages.

The proof follows the template of the proof from [9] but makes crucial use of Lemma 2. We use a variant of the reset lemma of [13].

Proof (Theorem 2). Let HF be a collision-resistant family of functions. Let $z(\cdot)=\mathrm{HF} . \mathrm{ol}(\cdot)+\mathrm{DE} . \operatorname{pkl}(\cdot)+\mathrm{DE} . \mathrm{pl}(\cdot)$. Let $n(\cdot)=2 z(\cdot)$. Let A be the adversary defined in Fig. 4. We should emphasize that the hash function here is not being applied element-wise, but to the ciphertext vector as a whole. Here, DE.Vf is the verification algorithm provided by Lemma 2 for $D E$. We first note that $A \in \mathcal{A}^{\text {soa }}$. Indeed, $A$ is unpredictable due to the assumption that $2^{-D E \cdot m l(\cdot)}$ is negligible and the fact that messages in the message vector are independently and uniformly 


\begin{tabular}{|c|c|}
\hline $\begin{array}{l}\text { Algorithm A.cs }\left(1^{\lambda}\right) \\
k \stackrel{\$}{\leftarrow}\{0,1\}^{\mathrm{HF} \cdot \mathrm{kl}(\lambda)} ; \text { Return } k \\
\text { Algorithm A.msg }\left(1^{\lambda}\right) \\
\mathbf{m} \stackrel{\$}{\leftarrow}\left(\{0,1\}^{\mathrm{DE} \cdot \mathrm{ml}(\lambda)}\right)^{n(\lambda)} ; \text { Return m } \\
\text { Algorithm A.cor }{ }^{\mathrm{CoR}}\left(1^{\lambda}, \pi, p k, k, \mathbf{c}\right) \\
b[1] \ldots b[z(\lambda)] \leftarrow \operatorname{HF}\left(1^{\lambda}, k, \mathbf{c}\right)\|\pi\| p k \\
I \leftarrow\{2 j-1+b[j]: 1 \leq j \leq z(\lambda)\} \\
\overline{\mathbf{m}} \leftarrow \operatorname{CoR}(I) \\
\text { st } \leftarrow(\pi, p k, \mathbf{c}) \\
\operatorname{Return~st~}\end{array}$ & $\begin{array}{l}\frac{\text { Algorithm A.g }\left(1^{\lambda}, k, I, \mathbf{s t}, \overline{\mathbf{m}}\right)}{(\pi, p k, \mathbf{c}) \leftarrow \text { st }} \\
\text { If }(|\pi| \neq \mathrm{DE} \cdot \mathrm{pl}(\lambda) \text { or }|p k| \neq \mathrm{DE} \cdot \mathrm{pkI}(\lambda)) \\
\quad \text { then return } 0 \\
\text { If }|\mathbf{c}| \neq n(\lambda) \text { then return } 0 \\
\text { If }\left(\text { not DE.Vf }\left(1^{\lambda}, \pi, p k\right)\right) \text { then return } 0 \\
b[1] \ldots b[z(\lambda)] \leftarrow \mathrm{HF}\left(1^{\lambda}, k, \mathbf{c}\right)\|\pi\| p k \\
\text { If }(I \neq\{2 j-1+b[j]: 1 \leq j \leq z(\lambda)\}) \\
\quad \text { then return } 0 \\
\text { For all } i \in I \text { do } \\
\quad \text { If }\left(\mathrm{DE} . \operatorname{Enc}\left(1^{\lambda}, \pi, p k, \overline{\mathbf{m}}[i]\right) \neq \mathbf{c}[i]\right) \\
\quad \text { then return } 0 \\
\text { Return } 1 \\
\text { Algorithm A.f }\left(1^{\lambda}, \mathbf{m}\right) \\
\text { Return } 1\end{array}$ \\
\hline
\end{tabular}

Fig. 4. Adversary A for the proof of Theorem 2

distributed. It also satisfies the distinctness condition since $2^{-\mathrm{DE} \cdot \mathrm{ml}(\cdot)}$ is negligible and $n(\cdot)$ is a polynomial. Next we note that

$$
\operatorname{Pr}\left[\operatorname{REAL}_{\mathrm{DE}}^{\mathrm{A}}(\lambda)\right]=1
$$

for all $\lambda \in \mathbb{N}$. This follows from the description of $A$ and the completeness of the verifier. We will build adversaries $X$ and $C$ such that

$$
\operatorname{Pr}\left[\operatorname{IDEAL}_{\mathrm{DE}}^{\mathrm{A}, \mathrm{S}}(\lambda)\right] \leq 2^{-\mathrm{DE} \cdot \mathrm{ml}(\lambda) z(\lambda)}+\sqrt{\mathbf{A d v}_{\mathrm{DE}, z, \mathrm{C}}^{\mathrm{ccr}}(\lambda)+\mathbf{A d v}_{\mathrm{HF}, \mathrm{X}}^{\mathrm{cr}}(\lambda)}
$$

for all $\lambda \in \mathbb{N}$. But by the assumption that HF is CR and by Lemma 2, we have that the above probability is not negligibly close to 1 and hence

$$
\operatorname{Adv}_{\mathrm{DE}, \mathrm{A}, \mathrm{S}}^{\text {soa }}(\cdot)=1-\operatorname{Pr}\left[\operatorname{IDEAL}_{\mathrm{DE}}^{\mathrm{A}, \mathrm{S}}(\cdot)\right]
$$

is a function that is not negligible.

It may seem strange that security fails for A.f that always returns 1 , because this function does not leak anything about $\mathbf{m}$. What we are saying is that it is not possible to prove even this simple, intuitive claim, meaning to give a simulator for an adversary relative to this simple information function.

We proceed to prove (3). Given any $S$, we divide it in two parts, $S_{1}$ and $S_{2}$. $S_{1}$ is the execution until the point at which the subset that will be corrupted is chosen, and $S_{2}$ is the rest of the execution. We assume without loss of generality that $S_{1}$ forwards the coins to $S_{2}$, so $S_{2}$ is deterministic. This means we can view $\mathrm{S}$ as operating as follows:

Simulator $\mathrm{S}^{\mathrm{COR}}\left(1^{\lambda}, k\right)$

$\left(\mathrm{st}^{*}, I\right) \stackrel{\$}{\leftarrow} \mathrm{S}_{1}\left(1^{\lambda}, k\right) ; \overline{\mathbf{m}} \leftarrow \operatorname{CoR}(I) ;$ st $\leftarrow \mathrm{S}_{2}\left(1^{\lambda}, \mathrm{st}^{*}, \overline{\mathbf{m}}\right) ;$ Return st 
We now provide some intuition about why we expect the simulator to fail. We consider an experiment where we run $A \cdot \operatorname{cs}\left(1^{\lambda}\right)$ to get $k$, run $\mathrm{S}_{1}\left(1^{\lambda}, k\right)$ to get (st* $\left.{ }^{*} I\right)$, pick two, random vectors $\overline{\mathbf{m}}_{0}, \overline{\mathbf{m}}_{1}$ that are $\perp$ on positions not in $I$, and then run $\mathrm{S}_{2}$ twice, getting $\mathrm{st}_{0} \leftarrow \mathrm{S}_{2}\left(1^{\lambda}, \mathrm{st}^{*}, \overline{\mathbf{m}}_{0}\right)$ and $\mathrm{st}_{1} \leftarrow \mathrm{S}_{2}\left(1^{\lambda}, \mathrm{st}^{*}, \overline{\mathbf{m}}_{1}\right)$. Parse $\mathrm{st}_{b}$ as $\left(\pi_{b}, p k_{b}, \mathbf{c}_{b}\right)$ for $b=0,1$. If $\mathrm{st}_{0} \neq \mathrm{st}_{1}$ then, because $I$ is the same in both cases, we have $\left(\pi_{0}, p k_{0}\right)=\left(\pi_{1}, p k_{1}\right)$ and thus $\mathbf{c}_{0} \neq \mathbf{c}_{1}$, leading to a collision for $\operatorname{HF}\left(1^{\lambda}, k, \cdot\right)$. So assume $\operatorname{st}_{0}=\mathrm{st}_{1}=(\pi, p k, \mathbf{c})$. If both runs make the game return true then by definition of A.g we have DE.Enc $\left(1^{\lambda}, \pi, p k, \overline{\mathbf{m}}_{0}[I]\right)=$ $\mathbf{c}[I]$ and DE.Enc $\left(1^{\lambda}, \pi, p k, \overline{\mathbf{m}}_{1}[I]\right)=\mathbf{c}[I]$. This is highly unlikely if the function DE.Enc $\left(1^{\lambda}, \pi, p k, \cdot\right)$ is injective. So the only way the simulator can hope to succeed is pick $\pi, p k$ so that this function is highly non-injective. But A.g is running the verifier so if the simulator tries this, A.g is likely to return 0 by Lemma 2 . In [8] we formalize the above intuition and establish (3) via the reset lemma.

InDistinguishabiLity-BASED SOA. Theorem 2 rules out SOA-secure D-PKE under a simulation-style definition. A natural question is whether SOA-secure D-PKE may be achieved under a weaker definition, in particular an indistinguishability style one. Indeed, for R-PKE, SOA-security definitions in both styles have been made and investigated, and the indistinguishability style is easier to achieve $[12,17,18,31]$. The difficulty is that for D-PKE it is not clear how to give a meaningful indistinguishability style definition of SOA-security. For R-PKE, the indistinguishability definition involves conditional re-sampling of the un-opened messages. In the D-PKE case we cannot provide the un-opened messages in the distinguishing test, since the adversary could easily win by reencrypting to check versus the ciphertexts. It is not clear to us what could be done instead. Additionally, even for R-PKE, re-sampling is rarely polynomial time so either we consider security for a very limited set of distributions or we have a non-polynomial time game, and both choices have problems. Defining some achievable notion of SOA-secure D-PKE is an interesting open problem.

\section{Does SU Security Imply MU Security?}

We now define mIND, the multi-key version of IND security, and show a separation between the two notions by showing the existence of a D-PKE scheme that is IND-secure but not mIND-secure.

mIND SECURITY. Let DE be a D-PKE scheme. An mIND adversary A specifies a common-state generation algorithm A.cs, a message-generation algorithm A.msg and a guessing algorithm A.g, all PT. On input $1^{\lambda}$, algorithm A.cs generates state information st that will be passed to both A.msg and A.g. Algorithm A.msg, on input $1^{\lambda}$, st returns a pair $\left(\mathbf{m}_{0}, \mathbf{m}_{1}\right)$ of $A \cdot n u(\lambda)$ by $A \cdot n m(\lambda)$ matrices over $\{0,1\}^{\mathrm{DE} \cdot \mathrm{ml}(\lambda)}$, where A.nu is the number-of-users function associated to $A$ and A.nm is the number-of-messages function associated to $A$. It is required that for each $b, i$ the strings $\mathbf{m}_{b}[i, 1], \ldots, \mathbf{m}_{b}[i, \mathrm{~A} \cdot \mathrm{nm}(\lambda)]$, which are the messages encrypted under the public key $\mathbf{p k}[i]$ of user $i$, be distinct. (However, messages 


\begin{tabular}{|c|c|}
\hline MAIN mIND $\operatorname{DE}_{\mathrm{DE}}^{\mathrm{A}}(\lambda)$ & MAIN PRED $\operatorname{PA}_{\mathrm{A}}^{\mathrm{P}}(\lambda)$ \\
\hline st $\stackrel{\$}{\leftarrow}$ A.cs $\left(1^{\lambda}\right) ;\left(\mathbf{m}_{0}, \mathbf{m}_{1}\right) \stackrel{\$}{\longleftarrow}$ A.msg $\left(1^{\lambda}\right.$, st $)$ & $($ st,$m) \stackrel{\$}{\leftarrow} \mathrm{P}\left(1^{\lambda}\right)$ \\
\hline$\pi \stackrel{\$}{\leftarrow} \mathrm{DE} \cdot \operatorname{Pg}\left(1^{\lambda}\right) ; b \stackrel{\$}{\leftarrow}\{0,1\}$ & $\left(\mathbf{m}_{0}, \mathbf{m}_{1}\right) \stackrel{\$}{\longleftarrow} \mathrm{A} \cdot \mathrm{msg}\left(1^{\lambda}, \mathrm{st}\right)$ \\
\hline For $i=1$ to $\mathrm{A} \cdot \mathrm{nu}(\lambda)$ do & $\operatorname{Return}\left(\exists i, j, b: \mathbf{m}_{b}[i, j]=m\right)$ \\
\hline$(\mathbf{p k}[i], \mathbf{s k}[i]) \stackrel{\$}{\leftarrow} \mathrm{DE} \cdot \mathrm{Kg}\left(1^{\lambda}, \pi\right)$ & \\
\hline $\begin{array}{l}\text { For } j=1 \text { to } \operatorname{A} \cdot \operatorname{nm}(\lambda) \text { do } \\
\quad \mathbf{c}[i, j] \leftarrow \operatorname{DE} \cdot \operatorname{Enc}\left(1^{\lambda}, \pi, \mathbf{p k}[i], \mathbf{m}_{b}[i, j]\right)\end{array}$ & \\
\hline$b^{\prime} \stackrel{\$}{\leftarrow}$ A.g $\left(1^{\lambda}, \pi, \mathbf{p k}, \mathbf{s t}, \mathbf{c}\right)$ & \\
\hline Return $\left(b=b^{\prime}\right)$ & \\
\hline
\end{tabular}

Fig. 5. The mIND game used to define multi-user security of D-PKE scheme DE and the PRED game used to define unpredictability of adversary $A$

\begin{tabular}{|c|c|}
\hline$\overline{\mathrm{DE}} \cdot \operatorname{Pg}\left(1^{\lambda}\right)$ & $\overline{\mathrm{DE}} . \operatorname{Enc}\left(1^{\lambda},\left(\pi, p k^{*}\right), p k, m\right)$ \\
\hline $\begin{array}{l}\pi \stackrel{\$}{\leftarrow} \mathrm{DE} \cdot \operatorname{Pg}\left(1^{\lambda}\right) \\
\left(p k^{*}, s k^{*}\right) \stackrel{\$}{\leftarrow} \mathrm{DE} \cdot \operatorname{Kg}\left(1^{\lambda}, \pi\right) \\
\text { Return }\left(\pi, p k^{*}\right)\end{array}$ & $\begin{array}{l}c \leftarrow \operatorname{DE} . \operatorname{Enc}\left(1^{\lambda}, \pi, p k, m\right) \\
c^{*} \leftarrow \operatorname{DE} . \operatorname{Enc}\left(1^{\lambda}, \pi, p k^{*}, m\right) \\
\text { Return }\left(c, c^{*}\right)\end{array}$ \\
\hline$\overline{\mathrm{DE}} . \operatorname{Kg}\left(1^{\lambda},\left(\pi, p k^{*}\right)\right)$ & DE. $\operatorname{Dec}\left(1^{\lambda},\left(\pi, p k^{*}\right), s k,\left(c, c^{*}\right)\right)$ \\
\hline 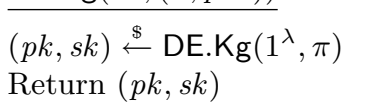 & $m \leftarrow$ DE.Dec $\left(1^{\lambda}, \pi, s k, c\right) ;$ Return $m$ \\
\hline
\end{tabular}

Fig. 6. D-PKE scheme $\overline{\mathrm{DE}}$ constructed from D-PKE scheme DE

may repeat across columns, meaning the same message may be encrypted under different public keys.)

The $\operatorname{mIND}_{\mathrm{DE}}^{\mathrm{A}}(\lambda)$ game associated with $\mathrm{DE}$ and adversary $\mathrm{A}$ is described on the left of Fig. 5. We define the advantage of $A$ via $\operatorname{Adv}_{\mathrm{DE}, \mathrm{A}}^{\min }(\lambda)=2$. $\operatorname{Pr}\left[\operatorname{mIND}_{\mathrm{DE}}^{\mathrm{A}}\left(1^{\lambda}\right)\right]-1$ for all $\lambda \in \mathbb{N}$. We let $\operatorname{Adv}_{\mathrm{A}, \mathrm{P}}^{\text {pred }}(\lambda)=\operatorname{Pr}\left[\operatorname{PRED}_{\mathrm{A}}^{\mathrm{P}}(\lambda)\right]$ for all $\lambda \in \mathbb{N}$, where game PRED is in the middle in Fig. 5. We say that $A$ is unpredictable if $\mathbf{A d}_{\mathbf{A}, \mathrm{P}}^{\text {pred }}(\cdot)$ is negligible for all $\mathrm{P}$. If $\mathcal{A}$ is a class (set) of adversaries then we say that $\mathrm{DE}$ is $\operatorname{mIND}[\mathcal{A}]$-secure if $\mathbf{A d v}_{\mathrm{DE}, \mathrm{A}}^{\operatorname{mind}}(\cdot)$ is negligible for all $\mathrm{A} \in \mathcal{A}$. It is convenient to view $\operatorname{mIND}[\mathcal{A}]$ as a set, so that $\mathrm{DE} \in \operatorname{mIND}[\mathcal{A}]$ iff $\mathrm{DE}$ is $\operatorname{mIND}[\mathcal{A}]$-secure. If A.cs always returns $\varepsilon$ then we say that $\mathrm{A}$ has trivial initial state and we may refer to $\mathrm{A}$ as a two-stage adversary. Let $\mathcal{A}_{2}^{\mathrm{m}}$ be the class of all PT, 2-stage unpredictable uniform adversaries, and for any polynomial $n: \mathbb{N} \rightarrow \mathbb{N}$ let $\mathcal{A}_{2, n}^{\mathrm{m}}$ be the class of all $\mathrm{A} \in \mathcal{A}_{2}^{\mathrm{m}}$ for which $\mathrm{A} . \mathrm{nu}=n$. Then security for $n$ users is captured by $\operatorname{mIND}\left[\mathcal{A}_{2, n}^{\mathrm{m}}\right]$ and security for any number of users is captured by $\operatorname{mIND}\left[\mathcal{A}_{2}^{\mathrm{m}}\right]$.

In the case of IND we had four variants, depending on whether adversaries were uniform or non-uniform and whether we were in the SM or the ROM. For simplicity, we address mIND in the uniform, SM case. The separation extends to the other three cases. Thus, below, the understanding is that IND, $\mathrm{mIND}_{n}, \mathrm{mIND}$ refer, respectively, to $\operatorname{IND}\left[\mathcal{A}_{2}^{\mathrm{sm}-\mathrm{u}}\right], \operatorname{mIND}\left[\mathcal{A}_{2, n}^{\mathrm{m}}\right]$ and $\operatorname{mIND}\left[\mathcal{A}_{2}^{\mathrm{m}}\right]$. 
SEPARATION RESUlt. Our separation is based on the minimal assumption that some IND-secure D-PKE scheme exists, and is established by a somewhat curious case analysis. The proof of the following is in [8].

Theorem 3. Assume there exists an IND-secure D-PKE scheme. Then there exists a D-PKE scheme that is (1) IND-secure but (2) not $\mathrm{mIND}_{2}$-secure.

Proof (Theorem 3). We establish the theorem by considering two cases.

Case 1: There does not exist a D-PKE scheme that is $\mathrm{mIND}_{2}$-secure.

The assumption in the theorem statement says there exists a D-PKE scheme DE that is IND-secure. But the assumption made for Case 1 says that no DPKE scheme is $\mathrm{mIND}_{2}$-secure. So in particular $\mathrm{DE}$ is not $\mathrm{mIND}_{2}$-secure. This establishes the theorem trivially in this case.

Case 2: There exists a D-PKE scheme that is $\mathrm{mIND}_{2}$-secure.

Let DE be a D-PKE scheme that is $\mathrm{mIND}_{2}$-secure. We construct from it a D-PKE scheme $\overline{\mathrm{DE}}$ that is (1) IND-secure but (2) not $\mathrm{mIND}_{2}$-secure. This establishes the theorem in Case 2. Since either Case 1 or Case 2 must be true, we have established the theorem overall.

The D-PKE scheme $\overline{\mathrm{DE}}$ is shown in Fig. 6. The parameters of the new scheme include a public key $p k^{*}$ for the old scheme. The new encryption of a message $m$ under public key $p k$ consists of two encryptions of $m$ under the old scheme, one with $p k$ and the other with $p k^{*}$. Intuitively, (2) is true because if users 1 , 2 encrypt messages $m_{1}, m_{2}$ then the second components of their ciphertexts are equal iff $m_{1}=m_{2}$, allowing an adversary to detect whether or not $m_{1}=m_{2}$. On the other hand, (1) is true because $p k^{*}$ can be viewed as a key of a dummy second user in the old scheme. Encryption in the new scheme is then tantamount to encryption of $m$ under two independent keys of the old scheme, which is secure by the assumed $\mathrm{mIND}_{2}$-security of the old scheme. We now proceed to the details.

We first establish (2), that $\overline{\mathrm{DE}}$ is not $\mathrm{mIND}_{2}$-secure, via the following adversary $\mathrm{A} \in \mathcal{A}_{2,2}^{\mathrm{m}}$. Let $\mathrm{A} \cdot \operatorname{cs}\left(1^{\lambda}\right)$ return $\varepsilon$. Let $\mathrm{A} \cdot \mathrm{msg}\left(1^{\lambda}, \varepsilon\right)$ return 2 by 1 matrices $\left(\mathbf{m}_{0}, \mathbf{m}_{1}\right)$ defined via

$$
\mathbf{m}_{0}[1,1], \mathbf{m}_{0}[2,1], \mathbf{m}_{1}[1,1] \stackrel{\$}{\leftarrow}\{0,1\}^{\mathrm{DE} \cdot \mathrm{ml}(\lambda)} ; \mathbf{m}_{1}[2,1] \leftarrow \mathbf{m}_{1}[1,1] .
$$

Let $\mathrm{A} \cdot \mathrm{g}\left(1^{\lambda},\left(\pi, p k^{*}\right), \mathbf{p k}, \varepsilon, \overline{\mathbf{c}}\right)$ parse $\left(\mathbf{c}[i, 1], \mathbf{c}^{*}[i, 1]\right) \leftarrow \overline{\mathbf{c}}[i, 1]$ for $i=1,2$. If $\mathbf{c}^{*}[1,1]=\mathbf{c}^{*}[2,1]$ then it returns 1 else it returns 0. Then $\operatorname{Adv}_{\overline{\mathrm{DE}}, \mathrm{A}}^{\text {mind }}(\lambda) \geq$ $1-2^{-\mathrm{DE} \cdot \mathrm{ml}(\lambda)}$.

To establish (1), that $\overline{\mathrm{DE}}$ is IND-secure, let $\overline{\mathrm{A}} \in \mathcal{A}_{2}$. We will provide $\mathrm{A} \in \mathcal{A}_{2,2}^{\mathrm{m}}$ such that

$$
\operatorname{Adv}_{\overline{D E}, \bar{A}}^{\text {ind }}(\lambda) \leq \operatorname{Adv}_{\mathrm{DE}, A}^{\operatorname{mind}}(\lambda)
$$

for all $\lambda \in \mathbb{N}$. Then (1) follows from the assumption that $\mathrm{DE}$ is $\mathrm{mIND}_{2}$-secure. Let A.cs $=\bar{A}$.cs return $\varepsilon$. Let A.nm $=\bar{A}$.nm. Let A.nu = 2. Define A.msg and A.g as follows: 


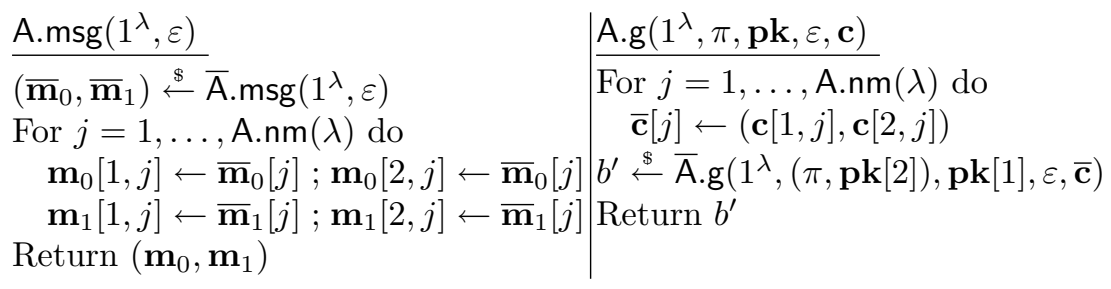

Then (5) follows.

We remark that the proof of Theorem 3 is non-constructive. It proves the existence of a scheme that is IND-secure but not $\mathrm{mIND}_{2}$-secure but does not put in our hands a concrete, specific example of such a scheme. This is because, although either Case 1 or Case 2 in the proof must be true, we do not know which. We also remark that our proof makes crucial use of the system parameters. Whether or not single and multi-user security are equivalent for D-PKE in the absence of system parameters is an interesting open question.

Acknowledgments. We thank Bjorn Tackmann, Peter Gazi and Adam O'Neill for valuable discussions. We thank the PKC 2015 reviewers for their valuable comments. Bellare was supported in part by NSF grants CNS-1228890 and CNS-1116800. Work done while Keelveedhi was at UCSD, supported in part by NSF grants CNS-1228890 and CNS-1116800.

\section{References}

1. Baudron, O., Pointcheval, D., Stern, J.: Extended notions of security for multicast public key cryptosystems. In: Welzl, E., Montanari, U., Rolim, J.D.P. (eds.) ICALP 2000. LNCS, vol. 1853, pp. 499-511. Springer, Heidelberg (2000)

2. Beaver, D.: Plug and play encryption. In: Kaliski Jr., B.S. (ed.) CRYPTO 1997. LNCS, vol. 1294, pp. 75-89. Springer, Heidelberg (1997)

3. Beaver, D., Haber, S.: Cryptographic protocols provably secure against dynamic adversaries. In: Rueppel, R.A. (ed.) EUROCRYPT 1992. LNCS, vol. 658, pp. 307-323. Springer, Heidelberg (1993)

4. Bellare, M., Boldyreva, A., Micali, S.: Public-key encryption in a multi-user setting: security proofs and improvements. In: Preneel, B. (ed.) EUROCRYPT 2000. LNCS, vol. 1807, p. 259. Springer, Heidelberg (2000)

5. Bellare, M., Boldyreva, A., O'Neill, A.: Deterministic and efficiently searchable encryption. In: Menezes, A. (ed.) CRYPTO 2007. LNCS, vol. 4622, pp. 535-552. Springer, Heidelberg (2007)

6. Bellare, M., Brakerski, Z., Naor, M., Ristenpart, T., Segev, G., Shacham, H., Yilek, S.: Hedged public-key encryption: how to protect against bad randomness. In: Matsui, M. (ed.) ASIACRYPT 2009. LNCS, vol. 5912, pp. 232-249. Springer, Heidelberg (2009)

7. Bellare, M., Desai, A., Pointcheval, D., Rogaway, P.: Relations among notions of security for public-key encryption schemes. In: Krawczyk, H. (ed.) CRYPTO 1998. LNCS, vol. 1462, p. 26. Springer, Heidelberg (1998) 
8. Bellare, M., Dowsley, R., Keelveedhi, S.: How secure is deterministic encryption? Cryptology ePrint Archive, Report 2014/376 (2014). http://eprint.iacr.org/2014/ 376

9. Bellare, M., Dowsley, R., Waters, B., Yilek, S.: Standard security does not imply security against selective-opening. In: Pointcheval, D., Johansson, T. (eds.) EUROCRYPT 2012. LNCS, vol. 7237, pp. 645-662. Springer, Heidelberg (2012)

10. Bellare, M., Fischlin, M., O'Neill, A., Ristenpart, T.: Deterministic encryption: definitional equivalences and constructions without random oracles. In: Wagner, D. (ed.) CRYPTO 2008. LNCS, vol. 5157, pp. 360-378. Springer, Heidelberg (2008)

11. Bellare, M., Hoang, V.T., Keelveedhi, S.: Instantiating random oracles via UCEs. In: Canetti, R., Garay, J.A. (eds.) CRYPTO 2013, Part II. LNCS, vol. 8043, pp. 398-415. Springer, Heidelberg (2013)

12. Bellare, M., Hofheinz, D., Yilek, S.: Possibility and impossibility results for encryption and commitment secure under selective opening. In: Joux, A. (ed.) EUROCRYPT 2009. LNCS, vol. 5479, pp. 1-35. Springer, Heidelberg (2009)

13. Bellare, M., Palacio, A.: GQ and schnorr identification schemes: proofs of security against impersonation under active and concurrent attacks. In: Yung, M. (ed.) CRYPTO 2002. LNCS, vol. 2442, p. 162. Springer, Heidelberg (2002)

14. Bellare, M., Rogaway, P.: Random oracles are practical: a paradigm for designing efficient protocols. In: Ashby, V. (ed.) ACM CCS 1993, pp. 62-73. ACM Press, November 1993

15. Bellare, M., Rogaway, P.: The security of triple encryption and a framework for code-based game-playing proofs. In: Vaudenay, S. (ed.) EUROCRYPT 2006. LNCS, vol. 4004, pp. 409-426. Springer, Heidelberg (2006)

16. Bellare, M., Waters, B., Yilek, S.: Identity-based encryption secure against selective opening attack. In: Ishai, Y. (ed.) TCC 2011. LNCS, vol. 6597, pp. 235-252. Springer, Heidelberg (2011)

17. Bellare, M., Yilek, S.: Encryption schemes secure under selective opening attack. Cryptology ePrint Archive, Report 2009/101 (2009). http://eprint.iacr.org/2009/ 101

18. Böhl, F., Hofheinz, D., Kraschewski, D.: On definitions of selective opening security. In: Fischlin, M., Buchmann, J., Manulis, M. (eds.) PKC 2012. LNCS, vol. 7293, pp. 522-539. Springer, Heidelberg (2012)

19. Boldyreva, A., Fehr, S., O'Neill, A.: On notions of security for deterministic encryption, and efficient constructions without random oracles. In: Wagner, D. (ed.) CRYPTO 2008. LNCS, vol. 5157, pp. 335-359. Springer, Heidelberg (2008)

20. Brakerski, Z., Segev, G.: Better security for deterministic public-key encryption: the auxiliary-input setting. In: Rogaway, P. (ed.) CRYPTO 2011. LNCS, vol. 6841, pp. 543-560. Springer, Heidelberg (2011)

21. Canetti, R., Dwork, C., Naor, M., Ostrovsky, R.: Deniable encryption. In: Kaliski Jr., B.S. (ed.) CRYPTO 1997. LNCS, vol. 1294, pp. 90-104. Springer, Heidelberg (1997)

22. Canetti, R., Feige, U., Goldreich, O., Naor, M.: Adaptively secure multi-party computation. In: 28th ACM STOC, pp. 639-648. ACM Press, May 1996

23. Canetti, R., Halevi, S., Katz, J.: Adaptively-secure, non-interactive public-key encryption. In: Kilian, J. (ed.) TCC 2005. LNCS, vol. 3378, pp. 150-168. Springer, Heidelberg (2005)

24. Damgård, I.B., Nielsen, J.B.: Improved non-committing encryption schemes based on a general complexity assumption. In: Bellare, M. (ed.) CRYPTO 2000. LNCS, vol. 1880, p. 432. Springer, Heidelberg (2000) 
25. Dodis, Y., Lee, P.J., Yum, D.H.: Optimistic fair exchange in a multi-user setting. In: Okamoto, T., Wang, X. (eds.) PKC 2007. LNCS, vol. 4450, pp. 118-133. Springer, Heidelberg (2007)

26. Dwork, C., Naor, M., Reingold, O., Stockmeyer, L.J.: Magic functions. Journal of the ACM 50(6), 852-921 (2003)

27. Fehr, S., Hofheinz, D., Kiltz, E., Wee, H.: Encryption schemes secure against chosen-ciphertext selective opening attacks. In: Gilbert, H. (ed.) EUROCRYPT 2010. LNCS, vol. 6110, pp. 381-402. Springer, Heidelberg (2010)

28. Fuller, B., O’Neill, A., Reyzin, L.: A unified approach to deterministic encryption: new constructions and a connection to computational entropy. In: Cramer, R. (ed.) TCC 2012. LNCS, vol. 7194, pp. 582-599. Springer, Heidelberg (2012)

29. Goldwasser, S., Micali, S.: Probabilistic encryption. Journal of Computer and System Sciences 28(2), 270-299 (1984)

30. Hemenway, B., Libert, B., Ostrovsky, R., Vergnaud, D.: Lossy encryption: constructions from general assumptions and efficient selective opening chosen ciphertext security. In: Lee, D.H., Wang, X. (eds.) ASIACRYPT 2011. LNCS, vol. 7073, pp. 70-88. Springer, Heidelberg (2011)

31. Hofheinz, D., Rupp, A.: Standard versus selective opening security: separation and equivalence results. In: Lindell, Y. (ed.) TCC 2014. LNCS, vol. 8349, pp. 591-615. Springer, Heidelberg (2014)

32. Kol, G., Naor, M.: Cryptography and game theory: designing protocols for exchanging information. In: Canetti, R. (ed.) TCC 2008. LNCS, vol. 4948, pp. 320-339. Springer, Heidelberg (2008)

33. Mironov, I., Pandey, O., Reingold, O., Segev, G.: Incremental deterministic publickey encryption. In: Pointcheval, D., Johansson, T. (eds.) EUROCRYPT 2012. LNCS, vol. 7237, pp. 628-644. Springer, Heidelberg (2012)

34. Nielsen, J.B.: Separating random oracle proofs from complexity theoretic proofs: the non-committing encryption case. In: Yung, M. (ed.) CRYPTO 2002. LNCS, vol. 2442, p. 111. Springer, Heidelberg (2002)

35. O'Neill, A.: Stronger security notions for trapdoor functions and applications. Ph.D. Thesis, Georgia Institute of Technology (2012)

36. Ouafi, K., Vaudenay, S.: Smashing SQUASH-0. In: Joux, A. (ed.) EUROCRYPT 2009. LNCS, vol. 5479, pp. 300-312. Springer, Heidelberg (2009)

37. Panjwani, S.: Tackling adaptive corruptions in multicast encryption protocols. In: Vadhan, S.P. (ed.) TCC 2007. LNCS, vol. 4392, pp. 21-40. Springer, Heidelberg (2007)

38. Peikert, C., Vaikuntanathan, V., Waters, B.: A framework for efficient and composable oblivious transfer. In: Wagner, D. (ed.) CRYPTO 2008. LNCS, vol. 5157, pp. 554-571. Springer, Heidelberg (2008)

39. Raghunathan, A., Segev, G., Vadhan, S.: Deterministic public-key encryption for adaptively chosen plaintext distributions. In: Johansson, T., Nguyen, P.Q. (eds.) EUROCRYPT 2013. LNCS, vol. 7881, pp. 93-110. Springer, Heidelberg (2013)

40. Ristenpart, T., Shacham, H., Shrimpton, T.: Careful with composition: limitations of the indifferentiability framework. In: Paterson, K.G. (ed.) EUROCRYPT 2011. LNCS, vol. 6632, pp. 487-506. Springer, Heidelberg (2011)

41. Ristenpart, T., Yilek, S.: When good randomness goes bad: Virtual machine reset vulnerabilities and hedging deployed cryptography. In: NDSS 2010. The Internet Society, February / March 2010

42. Wichs, D.: Barriers in cryptography with weak, correlated and leaky sources. In: Kleinberg, R.D. (ed.) ITCS 2013, pp. 111-126. ACM, January 2013 